\title{
Theoretical Overview: Light Ion Lessons, Heavy Ion Hopes
}

\author{
Sean Gavin \\ Department of Physics \\ Brookhaven National Laboratory \\ Upton, NY 11973
}

\begin{abstract}
Progress in understanding relativistic nuclear collisions is surveyed.
\end{abstract}

Invited talk at the

XXVI International Conference on High Energy Physics

Dallas, Texas, 6-12 August 1992

This manuscript has been authored under contract number DE-AC02-76CH00016 with the U.S. Department of Energy. Accordingly, the U.S. Government retains a non-exclusive, royalty-free license to publish or reproduce the published form of this contribution, or allow others to do so, for U.S. Government purposes.

\section{Guvieh}




\title{
THEORETICAL OVERVIEW: LIGHT ION LESSONS, HEAVY ION HOPES*
}

\author{
Sean Gavin \\ Department of Physics \\ Brookhaven National Laboratory \\ Upton, NY 11973
}

\begin{abstract}
Progress in understanding relativistic nuclear collisions is surveyed.
\end{abstract}

\section{INTRODUCTION}

Experiments using light ion beams of atomic masses $A \sim 30$ have been underway since 1986 at the Brookhaven AGS and the CERN SPS at the respective energies $\sqrt{s} \sim 5 \mathrm{~A} \mathrm{GeV}$ and $20 \mathrm{~A} \mathrm{GeV}$. The first truly heavy ion runs with a gold beam began this spring at the AGS. In this talk I will survey our progress towards an understanding of nuclear collision dynamics, focusing on those issues that are relevant to $\mathrm{Au}+\mathrm{Au}$ at the AGS (see Ref. [1] for a complete overview). In view of what we have already learned from the light ion data, I will argue that the prospects for producing matter at extreme density in these experiments are excellent.

\section{LIGHT ION LESSONS}

The aim of the AGS and SPS light ion programs is to ascertain the prospects for studying dense strongly interacting matter in heavy ion collisions. Important questions are:

1. How much energy does the projectile lose, i.e., what is the stopping power of the target [2]?

*This work was supported in part by contract number DE-AC02-76CH00016 with the U.S. Department of Energy.
2. How is this energy partitioned? Does the heavy ion system pass through a state near local thermal equilibrium?

3. How are strangeness and baryon number partitioned among the produced particles? Does the system approach chemical equilibrium?

4. What are the maximum energy and baryon densities? Where and when in the course of the collision are these conditions reached?

5. Are there any precursors of collective phenomena?

The term "collective phenomena" includes the sought-after possibility of quark gluon plasma formation but, more generally, refers to any interesting many body flow or particle production phenomena. Experience at the lower Bevalac energy $\sim 1 A \mathrm{GeV}$ indicates that evidence of collective flow can be obtained only through careful analysis of the heaviest $A A$ collisions [3].

\section{Extreme Models}

To illustrate the range of stopping power, equilibration, density, and collectivity, we consider the following extreme scenarios. In 
the independent fragmentation scenario, one supposes that the nuclear collision is a superposition of independent nucleon-nucleon, $N N$, subcollisions. Final state scattering of the primary nucleons and produced particles are entirely neglected. This scenario has been investigated using the first Monte Carlo event generators, such as the LUND/Fritiof model and early versions of VENUS [4]. These models yield a minimum of stopping power and allow for no collective behavior.

To characterize the opposite, fully collective extreme, one can imagine that the projectile and target participants stop each other completely to form a spherically symmetric fireball. Following an early thermal equilibrium stage, the fireball expands and rarefies until it's constituents cease to interact - the asymptotic particles are then formed. The evolution of the fireball has been studied extensively in the context of the Landau hydrodynamic formulation [5]. [N.B. Not all Landau models assume the spherical symmetry of a fireball [6].]

\section{Extreme Models vs. 'Jata}

Chapman and Gyulassy have pointed out that neither fireball nor independent fragmentation models stand up to a systematic comparison to the AGS light ion data [7]. They emphasized that fireball models give a proton rapidity distribution that disagrees seriously with measurements from BNL experiments E802 and E814, see refs. cited in [8]. The angular distribution of particles $d N / d \Omega$ from a spherically symmetric source is constant about the center of mass, so that the rapidity distribution is roughly

$$
\begin{aligned}
d N / d y_{\text {fireball }} & =(2 \pi d N / d \Omega) d \cos \theta_{\mathrm{cm}} / d y \\
& \propto\left\{\cosh \left(y-y_{\mathrm{cm}}\right)\right\}^{-1 / 2}
\end{aligned}
$$

where $y \approx y_{\mathrm{cm}}-\ln \left(\tan \theta_{\mathrm{cm}}\right)$, plus small corrections that depend on the particle mass. This

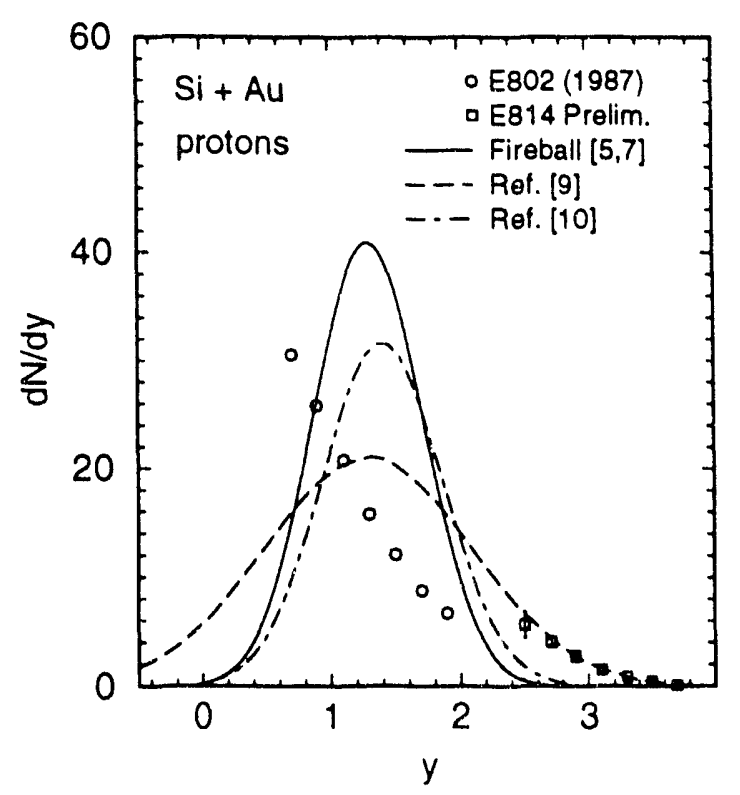

Figure 1. Measured proton rapidity density compared to fireball models from [7].

distribution is peaked near the participant center of mass rapidity, which is $y_{\mathrm{cm}} \sim 1.4$ for central $\mathrm{Si}+\mathrm{Au}$ (one counts as participants those target and projectile nucleons in the $\mathrm{di}$ rect path of the collision). Fig. 1 from [7] shows proton data from E802 and E814 compared with rapidity distributions of participant protons calculated for both the spherical fireball (including the mass corrections) and the variant models of ref. [9] and ref. [10]. The results are clearly above the data for $1<y<2$. Note that this discrepancy cannot be remedied by an appropriate treatment of spectator nucleons. In contrast, the rapidity spectra of secondaries from AGS and SPS are peaked at $y_{\mathrm{cm}}$, although the measured distributions are substantially more broad than the fireball shape [5].

The independent fragmentation picture is also wrong. While LUND model calculations agree with the proton rapidity density, Chapman and Gyulassy find that the pion $d N / d y$ are in serious disagreement with data [7]. The enhancement of strangeness production studied by E802 and E810 is also in conflict with independent fragmentation models. The $\mathrm{K}^{+}$ 
production per projectile nucleon in central $\mathrm{Si}+\mathrm{Au}$ is almost twice that in minimum bias $p+A u$ [11], and roughly four times the independent fragmentation model expectations [12]. Taken together, the various failures of independent fragmentation provide strong indications of the final state interactions omitted in that picture.

At the SPS, $J / \psi$ suppression [13] and $\phi$ enhancement [14] further support the need to include final state interactions and, moreover, suggest that high densities of secondary particles are achieved $[15,16]$. The $J / \psi$ suppression measured for oxygen and sulphur beams on uranium targets at $\sqrt{s}=20 \mathrm{~A} \mathrm{GeV}$ can be attributed to $N+J / \psi$ and meson $+J / \psi \rightarrow$ $D \bar{D}+X$ reactions, but not to $N+J / \psi$ interactions alone [15]. The meson densities needed to explain the data exceed $\sim 1 \mathrm{fm}^{-3}$ in models, as discussed by Satz in these proceedings.

\section{HADRONIC TRANSPORT MODELS}

To describe heavy ion dynamics consistently at AGS-SPS energies, one must address systems that are neither completely independent inor fully collective. Most phenomenological success has been in the context of an hadronic transport formulation. As in independent fragmentation one assumes that hadrons are formed in $N N$ subcollisions, only now one follows the trajectories of the particles and allows them to scatter. Rudimentary collective behavior associated with equilibration and flow can occur if scattering is sufficiently frequent.

In an hadronic formulation, one uses particle data to include various two body scattering processes, such as $\pi N \rightarrow \Delta, \pi N \rightarrow K \Lambda$, and $\pi \pi \rightarrow K \bar{K}$. However, cross sections for many reactions, e.g. $\Delta N \rightarrow \Delta N$ or $\pi \rho \rightarrow \omega \eta$, are not known and must essentially be guessed. In addition, formation times for the production of secondaries must be introduced to simulate uncertainty principle effects. To con- strain this vast ar.ay of parameters, one can turn to lepton-nucleus and hadron-nucleus data [17. 18]. Workers have found that $N, \pi$, and $K$ spectra depend primarily on a tractable subset of these parameters, so that the practical task of understanding heavy ion experiments is not as formidable as it might seem.

The most comprehensive phenomenological models applicable at AGS energies are the Monte Carlo cascade simulations RQMD [19] and ARC [20]. At SPS energies and above, one has a variety of models, such as HIJING discussed by $\mathrm{X} .-\mathrm{N}$. Wang in these proceedings (see also the papers by Werner, Amelin et al., Kawrakov et al., and Umar et al. in [1]). In principle, these models should describe all $p p$, $l A, h A$, and $A B$ data in their respective energy ranges with a single set of parameters. In addition, hosts of simpler but less comprehensive models have been employed to understand qualitative aspects of specific phenomena, e.g. $[25,26]$ discussed later.

At the higher RHIC and LHC energies, a large fraction of the particle production in nuclear collisions will result from semihard processes accessible via perturbative QCD methods [21]. To describe the initial stages of such collisions, Geiger has developed the partonic transport model presented in this session. The likelihood that such a QCD-based description can be applicable is one of the strongest motivations for moving to higher energies.

Transport Models us. Data

So far, the comprehensive cascade simulations are in reasonable agreement with experiment. Regarding the stopping power, proton rapidity distributions from ARC are in excellent accord with results from E814 at high rapidity and E802 at midrapidity [22]. The average rapidity loss per baryon is found to be $\sim 1.5$ units, as expected from simple multiple scattering extrapolations from $p A$ data. Such 
a rapidity loss is nearly sufficient to bring projectile participants of initial rapidity $y=3.4$ to rest in the center of mass on average there is significant stopping of the projectile. However, the target participants in the asymmetric $\mathrm{Si}+\mathrm{Au}$ collision are not accelerated into a fireball, cf. Fig. 1.

The measured strangeness enhancement discussed earlier constitutes circumstantial evidence of the scattering of secondaries necessary for thermal and chemical equilibration. The measured increase of the production of $K^{+}$and $\Lambda$ from $\mathrm{p}+\mathrm{p}$ to $\mathrm{p}+\mathrm{Au}$ to $\mathrm{Si}+\mathrm{Au}$ can be attributed to reactions such as $N \Delta \rightarrow K \Lambda N$, $\pi N \rightarrow K \Lambda$, and $\pi \pi \rightarrow K \bar{K}$. See the literature for detailed comparisons of RQMD [12, 23] and ARC $[20,22]$ to data.

There is however no direct quantitative indication of the densities, collectivity, or equilibration from the current light ion experiments. Signals of thermal equilibration are particularly subtle, since transverse momentum $p_{T}$ spectra from $p p \rightarrow \pi+X$ already appear thermal, $d \sigma / d p_{T}^{2} \sim \exp \left\{-\left(p_{T}^{2}+m_{\pi}^{2}\right)^{1 / 2} / T\right\}$, where $T$ is a temperature-like parameter. At the SPS, NA35 has measured an enhancement of charged particles relative to this $p p$ form for $p_{T}<200 \mathrm{MeV}$ in central $\mathrm{O}+\mathrm{Au}$ at midrapidity. Kataja and Ruuskanen pointed out that Bose enhancement can produce this effect, since pion densities at the SPS are likely $\sim 1 \mathrm{fm}^{-3}[24]$.

Transport models of nonequilibrium pion evolution indeed find a tendency towards this Bose enhancement, though the light ion systems are not fully equilibrated $[25,26]$. Nevertheless, there are several caveats: Theoretically, the full range of the Bose effect is extremely small - the difference between noninteracting and fully thermalized pion spectra in $\mathrm{O}+\mathrm{Au}$ is at most $20 \%$ [25]. Moreover, a nuclear enhancement of baryon resonance production must also contribute io the enhancement, since a larger low $p_{T}$ shift is measured at target rapidity $([25]$ and [26] only treat meson resonances). To estimate the significance of these various contributions at the $20 \%$ lev$\mathrm{el}$, it is necessary to include Bose statistics in more comprehensive cascade models that include baryons. Finally, the measurement of this small effect is quite intricate, since one must correct the charged particle spectra for misidentified electrons. SPS data with complete particle identification are forthcoming $[27]$.

As at the SPS, there is no consensus among AGS model builders as to which microscopic ingredients are necessary to explain the data. For example, ARC attributes the measured proton $p_{T}$ distribution to rescattering of the primary nucleons and baryon resonances [22], while RQMD requires the addition of a strong repulsive mean field interaction for baryons [23].

\section{HEAVY ION HOPES}

Comprehensive simulations like ARC and RQMD can be used to explore the spacetime dynamics of nuclear collisions. By following the trajectories of particles in model events, one can extract indications of the energy and baryon densities achieved in real collisions. However, the spacetime picture so obtained is consistent only if the computed final particle spectra agree with all available data.

ARC predictions for central $\mathrm{Au}+\mathrm{Au}$ at 11.6 A GeV beam energies indicate a gaussianshaped pion rapidity distribution with a peak value $d N / d y \approx 80$ for $\pi^{+}$at midrapidity, and a width that is roughly $60 \%$ larger than that of a fireball, cf. Eq. (1) [28]. RQMD pion distributions for $10 \mathrm{~A} \mathrm{GeV} \mathrm{Pb}+\mathrm{Pb}$ are roughly $\sim 40 \%$ higher [29].

\section{Collective Flow and High Densities?}

Collective flow has proven useful in probing the dynamics of nuclear collisions [3]. At the 
highest energies, hydrodynamic calculations suggest that a transverse collective expansion driven by pressure can reflect the equation of state of the underlying fluid, see, e.g. [30]. Despite early optimism, no clear transverse flow effects have been seen in light ion collisions. The most recent intensity interferometry measurements at BNL and CERN for pions and kaons imply transverse radii that are consistent with the projectile rms radii $[27,31]$.

In contrast to the lighter sysiems, ARC calculations suggest that transverse expansion can be substantial in $\mathrm{Au}+\mathrm{Au}$ at the AGS. To study the expansion, the distribution of the transverse radii for particles at the position of their last interaction is constructed. Pang, Schlagel and Kahana find a $60 \%$ increase in the average $\left\langle r_{\perp}\right\rangle$ of pions and protons compared to the rms radius of $\mathrm{Au}$. A relatively modest $\sim 30 \%$ increase is computed for $\mathrm{Si}+\mathrm{Au}$. [40] The impact of such a small $\mathrm{Si}+\mathrm{Au}$ increase on the interferometry signal is within the uncertainties in the analysis of this signal [32].

To illustrate the conditions that can be obtained in a central $\mathrm{Au}+\mathrm{Au}$ collision, Pang et al. follow the time evolution of the baryon density $\rho_{b}$ and average transverse momentum $\left\langle p_{T}\right\rangle$ for baryons in a test cell of $1 \mathrm{fm}$ radius in the interior of the collision volume. Fig. 2 shows the $\left\langle p_{T}\right\rangle-\rho_{b}$ trajectory of this cell for $\mathrm{Si}+\mathrm{Si}$ (squares) and $\mathrm{Au}+\mathrm{Au}$ (circles). The trajectory is traversed counterclockwise, with the plotting symbols spaced a time interval $1 \mathrm{fm} / \mathrm{c}$ apart in the cell's local rest frame. In essence, $\left\langle p_{T}\right\rangle^{2} / m$ measures the kinetic energy in the transverse degrees of freedom of baryons in the cell, where $m$ is the nucleon mass. If the nuclei simply fly through one another without any interaction, then geometry gives a peak density $\rho_{0} / \rho_{\mathrm{nm}} \sim 2 \gamma_{\mathrm{cm}} \sim 5.2$ for $\gamma_{\mathrm{cm}}=2.6$ and $\rho_{\mathrm{nm}}=0.16 \mathrm{fm}^{-3}$ for nuclear matter. For $\mathrm{Si}+\mathrm{Si}$ (and similarly for $\mathrm{Si}+\mathrm{Au}$ ), the baryons in the cell are compressed beyond $\rho_{0}$, but pass through the high density region very rapidly.
The trajectories for $\mathrm{Au}+\mathrm{Au}$ and $\mathrm{Si}+\mathrm{Si}$ are qualitatively different. The compression of baryons is greater in the heavier system. More importantly, the matter in the cell remains at the most extreme densities for a much longer time. If I add the dashed line to Fig. 2 as an "imaginary boundary" between the low and high energy density regions, then the $\mathrm{Au}+\mathrm{Au}$ spends 2-3 times longer in the high density region.

Observe the cusp shape at the highest density in $\mathrm{Au}+\mathrm{Au}$. I note that the trajectory at the cusp and afterward scales very nearly as $\left\langle p_{T}\right\rangle \propto \rho_{b}^{1 / 3}$, as expected for adiabatic expansion. $\mathrm{Si}+\mathrm{Si}$ does not exhibit this scaling, except perhaps in the final $\sim 2 \mathrm{fm} / \mathrm{c}$. Does this mean that matter in the interior of a $\mathrm{Au}+\mathrm{Au}$ collision approaches local equilibrium? More detailed model analyses are needed to see if the flow in ARC really is adiabatic and thermalized in this region. However, Sorge et al. find similar interior densities and strong indications of thermalization using RQMD [23].

If the cell really were in local equilibrium with its surroundings, then the temperature would be $T \approx 2\left\langle p_{T}\right\rangle^{2} / \pi m$. In a static, equilibrium system, the dasher curve would roughly correspond to the QCD phase boundary, assuming a first order chiral restoration transition at $T_{c}=200 \mathrm{MeV}$ for $\rho_{b}=0$. Of course, this cell is centrally located, and one expects the density to be higher there than in the periphery. Furthermore, the dynamics of the collision might proceed differently, e.g., if quark gluon plasma were to form. Nevertheless, I emphasize these rather conservative models suggest that $\mathrm{Au}+\mathrm{Au}$ can produce exactly the conditions that we hope for!

\section{Collective Particle Production?}

If the conditions in real $\mathrm{Au}+\mathrm{Au}$ experiments are as dramatic as simulations suggest, then one can expect the occurrence of nov- 


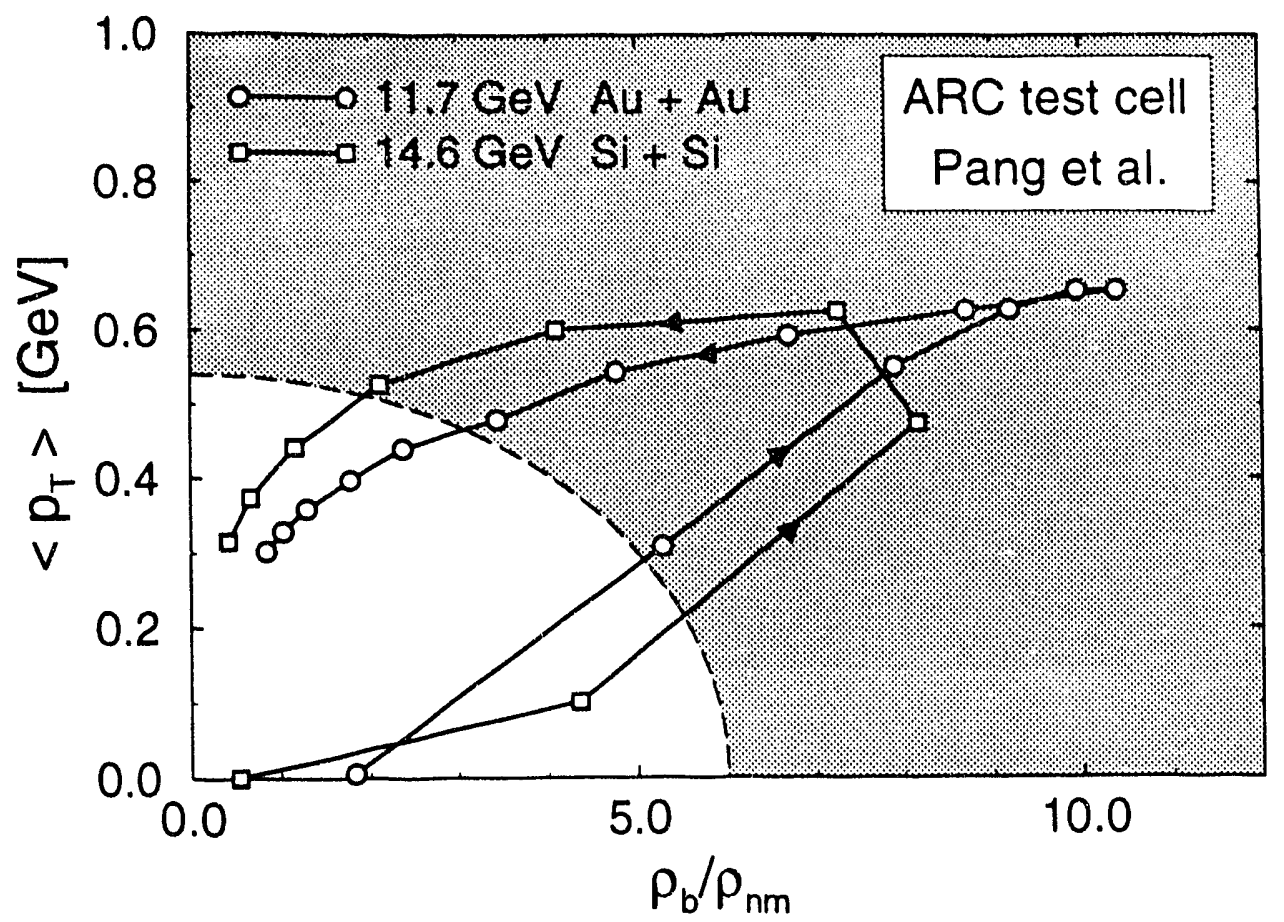

Figure 2. Time evolution of density and $\left\langle p_{T}\right\rangle$ for baryons in a test cell from ARC [28].

el collective phenomena associated with quark gluon plasma formation. Such phenomena may appear as a discrepancy between hadronic transport model predictions and data. The challenge will then be to prove that this discrepancy cannot be accounted for within the uncertainties in the models.

Where do we look for such discrepancies and what can we learn from them? One persistant puzzle concerns the order of the chiral restoration transition in QCD. Wilczek [33] has stressed that recent lattice results for two light flavors [34] are consistent with a second order transition. However, the latest simulations with smaller lattice spacing exhibit behavior tantalizingly close to that of a first order transition [35]. In view of these ambiguous results, let us ask if there are observables in heavy ion collisions that can depend on the nonequilibrium dynamics of the chiral transition.

Many authors have speculated that the chiral transition can enhance baryon-antibaryon pair production. Chiral restoration can con- tribute to this effect, e.g., by enhancing quarkantiquark pairs due to plasma formation, and by reducing the $\pi \pi \rightarrow N \bar{N}$ threshold as the in-medium hadron masses tend to zero. [Color rope formation [36] and minijet production can also enhance baryon pairs, but these mechanisms do not contribute at AGS energies.] Interestingly, DeGrand and Ellis, Heinz and Kowalski have argued by analogy with the cosmological Kibble mechanism that $N \bar{N}$ pairs can be enhanced in the Skyrme model due to the non-trivial topological structure induced by the formation of metastable chiral domains [37]. This mechanism explicitly depends on both the order of the transition and its nonequilibrium nature in $A A$ collisions.

It is extremely interesting that antiproton formation can depend on the order of the chiral transition; other chiral probes are discussed in [38]. One potential problem with the $\bar{p}$ probe is that annihilation may wipe out any initial excess. The mean free path for $\bar{p}$ 's is $\approx\left\{\rho_{b} \sigma_{a}\right\}^{-1}<0.3 \mathrm{fm}$, since the annihilation cross section is $\sigma_{a} \sim 40-100 \mathrm{mb}$ and the den- 
sity can exceed $\rho_{b} \sim 1 \mathrm{fm}^{-3}$.

In the absence of any collective production, however, we expect antiproton production to be suppressed [39]. Specifically, Gyulassy, Plümer, Venugopalan and myself predicted that annihilation reduces the $\bar{p} / p$ ratio in $A A$ relative to $p p$ collisions.

ARC calculations [40] indeed find that annihilation modifies the dependence of $\bar{p}$ production on $A$ and centrality in accord with the AGS light ion data discussed by Kumar in these proceedings. However, there are uncertainties in the cascade description of $\bar{p} \mathrm{dy}-$ namics at $\sqrt{s}=5 \mathrm{~A} \mathrm{GeV}$. The production in $N N$ subcollisions is near threshold and, consequently, is very sensitive to the treatment of rescattering. More significant is the uncertainty in the medium effects in the annihilation. Kahana et al. point out that the large $\sigma_{a}$ can be shielded, since the interacting $\bar{p}$ can scatter in the medium before their antiquark content is annihilated.

These large uncertainties leave ample room for enhancement in light ion collisions. To exhibit possible enhancement effects in $\mathrm{Au}+\mathrm{Au}$, it is useful to study the centrality dependence of $\bar{p}$ and $p$ production [41]. The more central is the collision, the larger is the baryon density and, correspondingly, the greater is the annihilation. On the other hand, the higher energy density in central collisions can lead to chiral restoration in a larger fraction of the collision volume, which in turn can produce more initial baryon pairs. ARC calculations find that $\bar{p} / p$ at midrapidity can decrease by a factor of $\sim 5$ as the impact parameter is varied from 10 $\mathrm{fm}$ to 0 [40]. It would be extremely interesting if this year's measurements reveal an enhancement relative to these predictions!

I am grateful to Yang Pang, Tom Schlagel, and Sid Kahana for furnishing me with ARC results, and to M. Bloomer, S. Chapman, S. Gottlieb, M. Gyulassy, T. Matsui, R. Pisarski, W. Schaffer, and W. Zajc for stimulating discussions.

\section{REFERENCES}

1. Proc. Quark Matter '91 Cunf., T.C. Awes, F.E. Obenshain, F. Plasil, M.R. Strayer and C.Y. Wong eds., Nucl. Phys. A544 (1992).

2. Here, "stopping power" refers to the momentum distribution of baryons, rather than the spacetime evolution of baryons.

3. H.H. Gutbrod, A.M. Postkanzer and H.G. Ritter, Rep. Prog. Phys. 52, 1267 (1989).

4. B. Anderson et al., Nucl. Phys. B281, 289 (1987); J.A. Casado et al., Z. Phys. C33, 541 (1987); K. Werner, Phys. Lett. 208B, 502 (1988).

5. E. Schnedermann, J. Sollfrank, and U. Heinz, TPR-92-29, to appear in Proc. NATO Advanced Study Institute on Particle Production in Highly Excited Matter, Il Ciocco, Italy, July 12-24 1992, H.H. Gutbrod ed., Plenum Press, New York 1992.

6. U. Ornik, F.W. Pottag and R.M. Weiner, in Hadronic Matter in Collision 1988, P.A. Carruthers and J. Rafelski eds., Singapore, 1989, p. 310; R. Venugopalan and M. Prakash, Phys. Rev. C41, 221 (1990); R. Venugopalan, PhD Thesis, Stony Brook (1992), unpublished.

7. S. Chapman and M. Gyulassy, Phys. Rev. Lett. 67, 1210 (1991); Phys. Rev. C45, 2952 (1992).

8. S. Nagamiya in [1], p. $5 \mathrm{c}$.

9. J. Stachel and P. Braun-Munzinger, Phys. Lett. B216, 1 (1989).

10. G. Brown et al., Phys. Rev. C43, 1181 (1991); C. M. Ko et al., Phys. Rev. Lett. 66, 2577 (1991); 67, 1811 (1991).

11. T. Abbott et al (E802), Phys. Rev. Lett. 66, 1567 (1991); K. J. Foley (E810) in [1], p. $335 \mathrm{c}$. 
12. R. Mattiello et al., Phys. Rev. Lett. 63, 1459 (1989).

13. C. Baglin et al., Phys. Lett. B270, 105 (1991), and refs. therein.

14. R. Ferreira (NA38) in [1], p. 497c; M.A. Mazzoni (NA34) in [1], p. 623c.

15. S. Gavin and R. Vogt, Nucl. Phys. B345, 104 (1990); R. Vogt in [1], p. 615c; S. Gavin, H. Satz, R.L. Thews, and R. Vogt, in progress.

16. P. Koch, U. Heinz, and J. Pisut, Phys. Lett. B243, 149 (1990); F. Grassi and H. Heiselberg in [1], p. 619c.

17. A. Bialas and M. Gyulassy, Nucl. Phys. B291, 793 (1987).

18. M. Gyulassy and M. Plümer, Nucl. Phys. B346, 1 (1990).

19. H. Sorge, H. Stöcker, and W. Greiner, $A n$ n. Phys. (N.Y.) 192, 266 (1989).

20. Y. Pang, T.J. Schalgel, and S.H. Kahana, Phys. Rev. Lett. 68, 2743 (1992).

21. K. Kajantie et al. Phys. Rev. Lett. 59, 2517 (1987); J.P. Blaizot and A.H. Mueller, Nucl. Phys. B289, 847 (1987); K.J. Eskola et al. Nucl. Phys. B323, 37 (1989).

22. T.J. Schlagel, S.H. Kahana, and Y. Pang, BNL-47608, to appear Phys. Rev. Lett. (1992).

23. H. Sorge et al., Phys. Rev. Lett. 68, 286 (1992); A. Jahns et al., Phys. Rev. Lett. 68, 2895 (1992).

24. M. Kataja and P.V. Ruuskanen, Phys. Lett. B243, 181 (1990); P. Gerber et al, Phys. Lett. B246, 513 (1990).

25. S. Gavin and P.V. Ruuskanen, Phys. Lett. B262, 326 (1991); S. Gavin in [1], p. 459c.

26. H.W. Barz, P. Danielewicz, H. Schulz, and G.M. Welke, MSUCL-805 (1992); G.M. Welke and G. Bertsch, Phys. Rev. C45, 1403 (1992).
27. M. Sarabura (NA44 Collab.), in [1], p. $125 \mathrm{c}$.

28. T.J. Schlagel, Y. Pang, and S.H. Kahana, in preparation.

29. H. Sorge et al., Phys. Lett. B243, 7 (1990).

30. G. Baym et al., Nucl. Phys. A407, 541 (1983); M. Kataja et al. Z. Phys. C55, 153 (1992).

31. W.A. Zajc (E802 Collab.), in [1], p. 237c; P. Seyboth (NA35 Collab.), in [1], p. 293c.

32. M. Gyulassy and S.S. Padula, in [1], p. 537 c; Phys. Lett. B217, 181 (1989).

33. F. Wilczek, IASSNS-HEP-91/65, (1991); R. Pisarski and F. Wilczeck, Phys. Rev. D29, 338 (1984).

34. F.R. Brown et al., Phys. Rev. Lett. 65, 2491 (1990).

35. C. Bernard et al., IUHET-222, (1992); N.H. Christ in these proceedings.

36. J. Knoll, Z. Phys. C38, 187 (1988); H. Sorge et al., Phys. Lett. B289, 6 (1992).

37. T.A. DeGrand, Phys. Rev. D30, 2001 (1984); J. Ellis et al., Phys. Lett. B233, 223 (1989).

38. Y. Takahashi and S. Nagamiya, Nucl. Phys. A525, 623c (1991); J.D. Bjorken, Int. J. Mod. Phys. A7, 4189 (1992).

39. S. Gavin, M. Gyulassy, M. Plümer and R. Venugopalan, Phys. Lett. B234, 175 (1990).

40. S.H. Kahana, Y. Pang, T.J. Schlagel and C.B. Dover, in preparation.

41. S. Gavin Nucl. Phys. A525, 459c (1991). 

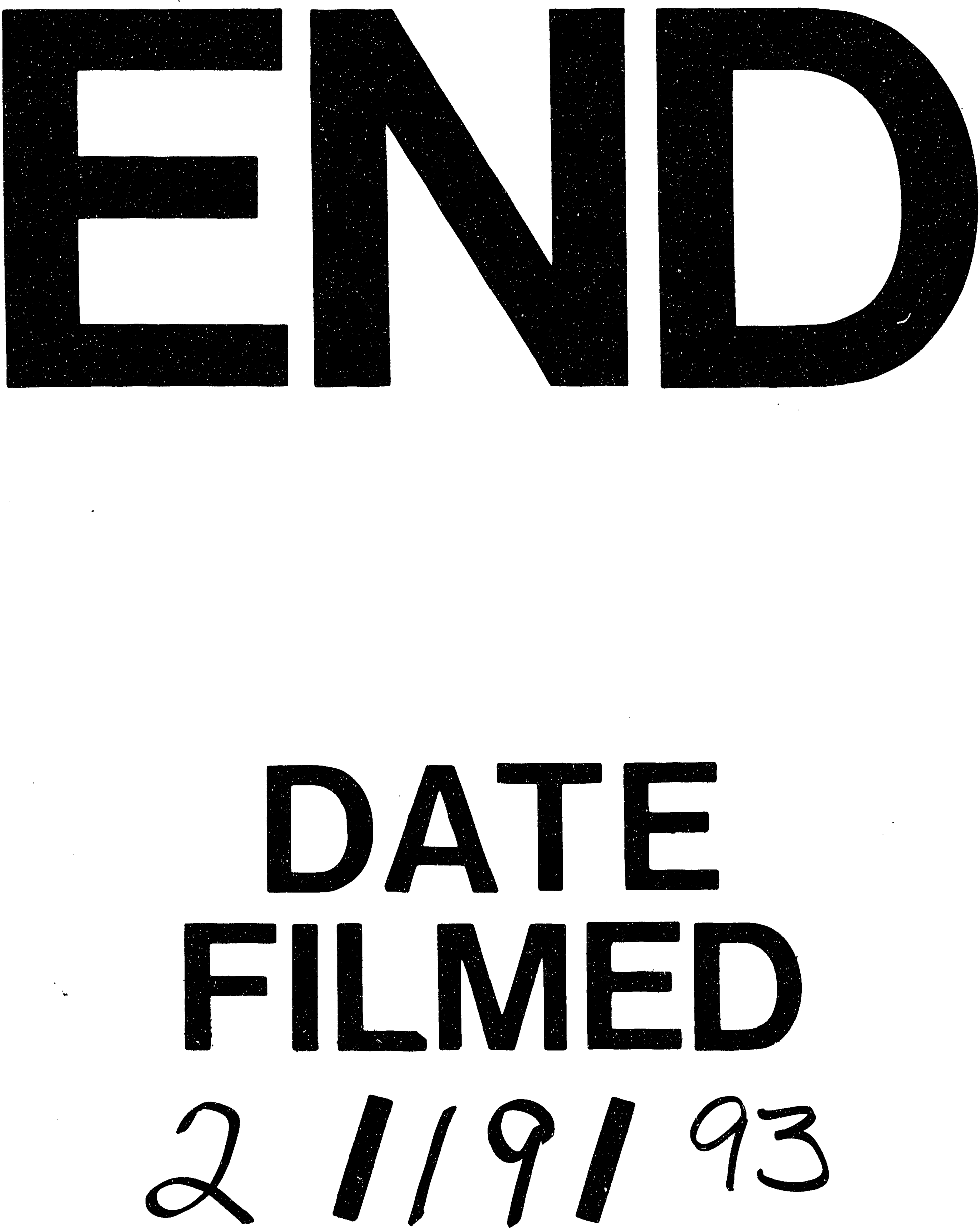


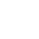

